\title{
Pseudo-admittance Bilateral Telemanipulation with Guidance Virtual Fixtures
}

\author{
Jake J. Abbott* Allison M. Okamura ${ }^{\dagger}$ \\ Department of Mechanical Engineering \\ The Johns Hopkins University
}

\begin{abstract}
We present a novel bilateral telemanipulation control system called Pseudo-admittance, which is designed to mimic admittance control on systems where the master is an impedance-type robot. Desirable system properties include steady-hand tremor attenuation and quasi-static transparency. The controller can also be modified to include virtual fixtures that provide guidance, while leaving ultimate control of the system with the operator. The properties of the system are verified through simulations and experiments. Pseudo-admittance control has potential benefits for tasks that require better-than-human levels of precision, as well as with systems which are typically run under rate control. Guidance virtual fixtures could be used as task macros - potentially increasing both speed and precision on structured tasks that require direct human control.
\end{abstract}

CR Categories: H.5.2 [Information Interfaces and Presentation]: User Interfaces-Haptic I/O; I.2.8 [Artificial Intelligence]: Problem Solving, Control Methods, and Search-Control Theory; I.2.9 [Artificial Intelligence]: Robotics-Manipulators; I.2.9 [Artificial Intelligence]: Robotics-Operator Interfaces

Keywords: virtual fixtures, virtual mechanisms, telemanipulation, teleoperation, rate control, admittance control, force control

\section{INTRODUCTION}

Bilateral telemanipulation refers to systems where a human operator manipulates a master robotic device, and a slave robotic device emulates the behavior of the master, with some form of haptic (force and/or tactile) feedback to the operator. In this paper, we present a novel bilateral telemanipulation scheme that we call Pseudo-admittance. Admittance control, where the velocity of the robot is proportional to the applied force, is typically implemented on admittance-type robots, which can be modeled as nonbackdrivable with velocity-source actuators. Pseudo-admittance control mimics admittance control on telemanipulation systems with impedance-type masters. Robots of the impedance type are backdrivable - with low inertia, low friction, and force-source actuators. Since Pseudo-admittance control does not require admittance-type hardware, it can be overlayed on existing impedance-type telemanipulators designed for transparency. The controller can then be turned on and off as desired. Our method generalizes to systems with impedance- or admittance-type slaves.

When commanding the slave robot through free space, Pseudoadmittance control results in a slave velocity that is roughly lin-

\footnotetext{
*e-mail: jabbott@ethz.ch. Jake Abbott is now with the Institute of Robotics and Intelligent Systems, ETH Zurich, Switzerland.

†e-mail: aokamura@jhu.edu. This work is supported by NSF grants \#ITR-0205318 and \#IIS-0347464.

Symposium on Haptic Interfaces for

Virtual Environment and Teleoperator Systems 2006

March 25 - 26, Alexandria, Virginia, USA

1-4244-0226-3/06/\$20.00 O2006 IEEE
}

early proportional to the user's applied force. When the robot contacts an environment, the controller feels roughly like force control. Pseudo-admittance control is designed to have asymmetries that provide desirable steady-hand characteristics, in that highfrequency movements of the master are attenuated at the slave, but still allow for high-fidelity force feedback from the slave to the master. Pseudo-admittance control also exhibits quasi-static transparency. That is, the system has static equilibrium points if and only if there is perfect (scaled) position correspondence between the master and the slave, and the (scaled) slave/environment interaction force is perfectly reflected to the user. In addition, this property is closely approximated when the system is moving slowly. Pseudoadmittance control can generate large slave/environment interaction forces, regardless of controller gains. Pseudo-admittance control has potential benefits on tasks that require better-that-human levels of precision. We are particularly interested in applications in robot-assisted surgery.

Pseudo-admittance control also has potential benefits for systems that are traditionally run under rate control, such as heavy hydraulic equipment [10]. Rate control is typically desirable when either the workspace of the slave is much larger than the workspace of the master, or the slave device has restrictive velocity saturation limits. If position control is used on systems with significant motion scaling, hand tremor and other undesirable movements of the user are amplified at the slave. Rate control also has potential benefits even without motion scaling, if the precision required at the slave manipulator is beyond the limits of the human user. In recent years, researchers have worked towards providing force feedback on telemanipulators under rate control. However, it is not obvious how best to add force feedback to rate-controlled systems, due to the kinematic discrepancies between the master and the slave. Methods to create transparent rate-controlled bilateral telemanipulation are reviewed in [12], where transparency is defined as accurately presenting the environment's impedance to the human user. An alternative is Naturally Transitioning Rate-to-Force Control [17], which acts like rate control when the slave is moving in free space, and acts like force control when the slave is constrained by an environment.

Positioning tasks are most intuitively accomplished using position control [8], and consequently, researchers have worked towards correcting the deficiencies in position control, as an alternative to rate control. Casals et al. [3] introduce a workspace-deformation method that increases precision at key locations in the workspace (at the expense of reducing precision elsewhere). Kontz and Book [9] present a method that provides an alternative to rate control for an application where rate control is typically used: the control of a hydraulic forklift. Pseudo-admittance control also provides a novel alternative to rate control that retains some of the benefits of traditional position control. Like [17], Pseudo-admittance control is also "naturally transitioning," in that it requires no controller switching event in the transition between free and constrained motion.

The structure of the Pseudo-admittance controller also lends itself to the implementation of guidance virtual fixtures (GVFs). GVFs assist the user in moving the slave manipulator along desired 

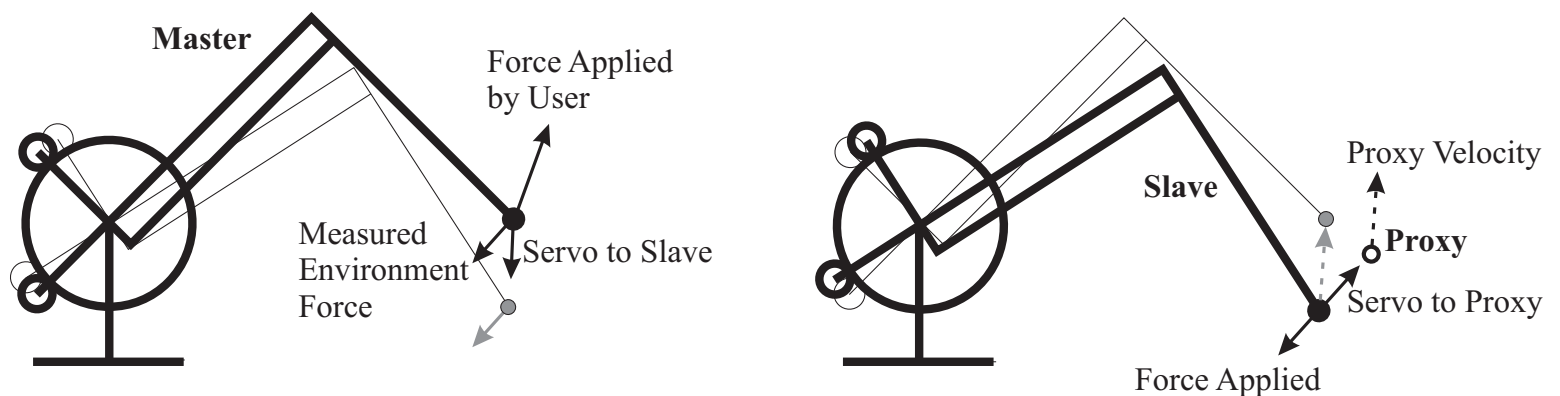

by Environment

Figure 1: Pseudo-admittance Bilateral Telemanipulation. Projections of master/slave manipulators in slave/master space are shown.

paths or surfaces. GVFs have been implemented on passive cooperative robotic systems (where the human and robot simultaneously act on a single end-effector) known as Cobots [13]. These GVFs act in a passive way in the sense that they are only able to restrict, and not generate, motion. It is also possible to implement similar GFVs using active admittance-type systems. Bettini et al. [2] implemented GVFs on the Johns Hopkins University Steady-Hand Robot, which is an admittance-type cooperative manipulator. In this paper, we extend these GVFs to telemanipulators with impedancetype masters. When implementing GVFs via Pseudo-admittance control, the user retains ultimate control to move the slave anywhere in the workspace. The user also experiences quasi-static transparency in both the constrained and the unconstrained directions. This property is unique among previous implementations of GVFs. One of the potential uses of GVFs under Pseudo-admittance control is as task-specific macros that would allow a user to quickly and safely conduct structured tasks.

\section{Pseudo-admittance Control}

Pseudo-admittance control is designed to mimic the following admittance-type control system:

$$
\dot{X}_{s}=\dot{X}_{m}=K_{a}\left(F_{h}+\gamma_{f} F_{e}\right)
$$

where $\dot{X}_{m}$ and $\dot{X}_{s}$ are the master and slave velocities, respectively, $F_{h}$ and $F_{e}$ are the applied human and slave/environment forces, respectively, $\gamma_{f}$ is a force scaling gain, and $K_{a}$ is a user-defined diagonal admittance gain matrix. A similar admittance control law (also known as proportional-velocity control) has previously been explored with the Johns Hopkins University Steady-Hand Robot [14]. The admittance control of (1) can be accomplished directly if both the master and slave devices are of the admittance type. However, many telemanipulators are of the impedance type, particularly those designed for robot-assisted surgery, such as the da Vinci Surgical System [7]; this motivates our work.

Pseudo-admittance bilateral telemanipulation is a proxy-based controller [18] that works as follows: a proxy exists in software, the slave robot servos to the proxy, the master servos to the slave, the measured environmental force $F_{e}$ is scaled and fed directly to the master, and the proxy moves as a function of the error between the master and the slave positions. The controller is illustrated in Fig. 1, and described in detail in the following.

\subsection{Control System Algorithm}

The master and slave devices we consider are assumed to be seriallink robots with dynamics

$$
M_{i}\left(\Theta_{i}\right) \ddot{\Theta}_{i}+N_{i}\left(\Theta_{i}, \dot{\Theta}_{i}\right)=\Upsilon_{a i}+J_{i}^{T}\left(\Theta_{i}\right) F
$$

where $\Theta_{i}$ is the vector of joint variables, $M_{i}$ is the positive-definite inertia matrix, $N_{i}$ is the vector containing Coriolis and centrifugal terms, as well as gravity effects and joint friction, $\Upsilon_{a i}$ is the vector of joint actuator forces/torques, and $J_{i}$ is the robot's velocity Jacobian, where $i=(m, s)$ for the master and slave devices. $F$ is the force vector that is externally applied to the robot end effector, expressed in the same frame as the Jacobian. For the master device $F=F_{h}$ is the force applied by the human, for the slave $F=F_{e}$ is the environmental force.

Using a linearizing and decoupling control law [5], also known as the computed-torque method, we assume a model

$$
\ddot{X}_{i}=F_{c i}+M_{x i}^{-1}\left(\Theta_{i}\right) F
$$

where $X_{i}$ is the Cartesian position of the robot, $M_{x i}$ is the Cartesian inertia matrix, and $F_{c i}$ is the Cartesian controller force vector, where $i=(m, s)$ for the master and slave devices.

We command the slave to servo to the proxy, using proportional control with velocity feedback:

$$
F_{c s}=K_{p s}\left(X_{p}-X_{s}\right)-K_{d s} \dot{X}_{s}
$$

$X_{p}$ is the position of the proxy, and $K_{p s}$ and $K_{d s}$ are the positivedefinite proportional and derivative control matrices. We will assume an isotropic servo controller, where $K_{p s}$ and $K_{d s}$ can be expressed as a scalar gain multiplied by an identity matrix.

We command the master to servo to the slave using proportionalderivative (PD) control. In addition, we feed forward a scaled version of the measured environmental force:

$$
F_{c m}=K_{p m}\left(X_{s}-X_{m}\right)+K_{d m}\left(\dot{X}_{s}-\dot{X}_{m}\right)+M_{x m}^{-1}\left(\Theta_{m}\right) \gamma_{f} F_{e}
$$

where $\gamma_{f}$ is the scalar force-scaling gain, and $K_{p m}$ and $K_{d m}$ are isotropic as before. The appearance of the Cartesian inertia matrix in (3) is an artifact of the linearizing and decoupling control law [5].

The proxy moves with the programmed dynamics

$$
\dot{X}_{p}=K_{a} F_{P D m}
$$

where $K_{a}$ is the positive-definite diagonal admittance gain matrix, and $F_{P D m}$ is the component of the user's applied force due to the master's PD servo controller:

$$
F_{P D m}=\hat{M}_{x m}\left(\Theta_{m}\right)\left[K_{p m}\left(X_{m}-X_{s}\right)+K_{d m}\left(\dot{X}_{m}-\dot{X}_{s}\right)\right]
$$

where $\hat{M}_{x m}$ is the estimate of $M_{x m}$. There may be cases where different admittance gains are desired in different directions of the workspace, but typically the admittance gain matrix will be chosen to be isotropic. 


$$
\begin{aligned}
{\left[\begin{array}{c}
\ddot{E}_{m} \\
\dot{E}_{m} \\
\ddot{X}_{s} \\
\dot{E}_{s} \\
\dot{X}_{p}
\end{array}\right]=} & {\left[\begin{array}{ccccc}
-K_{d m} & -K_{p m} & -K_{d s} & -K_{p s} & 0 \\
I & 0 & 0 & 0 & 0 \\
0 & 0 & -K_{d s} & -K_{p s} & 0 \\
K_{a} \hat{M}_{x m}\left(\Theta_{m}\right) K_{d m} & K_{a} \hat{M}_{x m}\left(\Theta_{m}\right) K_{p m} & I & 0 & 0 \\
-K_{a} \hat{M}_{x m}\left(\Theta_{m}\right) K_{d m} & -K_{a} \hat{M}_{x m}\left(\Theta_{m}\right) K_{p m} & 0 & 0 & 0
\end{array}\right]\left[\begin{array}{c}
\dot{E}_{m} \\
E_{m} \\
\dot{X}_{s} \\
E_{s} \\
X_{p}
\end{array}\right] } \\
+ & {\left[\begin{array}{cc}
-M_{x m}^{-1}\left(\Theta_{m}\right) & M_{x s}^{-1}\left(\Theta_{s}\right)-\gamma_{f} M_{x m}^{-1}\left(\Theta_{m}\right) \\
0 & 0 \\
0 & M_{x s}^{-1}\left(\Theta_{s}\right) \\
0 & 0 \\
0 & 0
\end{array}\right]\left[\begin{array}{c}
F_{h} \\
F_{e}
\end{array}\right] }
\end{aligned}
$$

We will be particularly interested in two error variables: the position error between the slave and the proxy $\left(E_{s}=X_{s}-X_{p}\right)$, and the position error between the master and slave $\left(E_{m}=X_{s}-X_{m}\right)$. The system dynamic equations, in terms of these error variables, are given by:

$$
\begin{aligned}
\ddot{X}_{m} & =K_{p m} E_{m}+K_{d m} \dot{E}_{m}+M_{x m}^{-1}\left(\Theta_{m}\right)\left(F_{h}+\gamma_{f} F_{e}\right) \\
\ddot{X}_{s} & =-K_{p s} E_{s}-K_{d s} \dot{X}_{s}+M_{x s}^{-1}\left(\Theta_{s}\right) F_{e} \\
\dot{X}_{p} & =-K_{a} \hat{M}_{x m}\left(\Theta_{m}\right)\left(K_{p m} E_{m}+K_{d m} \dot{E}_{m}\right)
\end{aligned}
$$

We will assume that $\hat{M}_{x m}$ is updated continuously for the purposes of the linearizing and decoupling controller, but in the proxy dynamics of (7) it will be updated at a rate that is slow relative to the other system dynamics. This is done for the purposes of ensuring stability (this topic is discussed in Section 2.2).

In order to analyze stability and characterize performance, we are interested in expressing our system with respect to the variables $\dot{E}_{m}, E_{m}, \dot{X}_{s}, E_{s}$, and $X_{p}$. The system written in this state-space form is given as (6).

\subsection{Stability}

To analyze the stability of (6), we must first begin by defining what stability means for our system. From an arbitrary initial condition, we would like the unforced system $\left(F_{h}=F_{e}=0\right)$ to come to rest $\left(\dot{X}_{m}, \dot{X}_{s}, \dot{X}_{p} \rightarrow 0\right)$ with no position error between the master and the slave $\left(E_{m} \rightarrow 0\right)$. We cannot characterize the stability of our system by the stability of some equilibrium state vector. For our system, the position of the proxy $X_{p}$ (and consequently the master and slave position) should move around the workspace in an unbounded fashion; there is no zero position to which we would like the unforced system to return.

The block-triangular structure of the state matrix in (6) allows us to consider the stability of the error system (consisting of just the first four states) independently from $X_{p}$. We would like this new system (when unforced) to have a stable equilibrium at its zero state vector. We would also like this system to be bounded-input/bounded-output (BIBO) stable; that is, we would like bounded external forces to lead to bounded master and slave velocities and bounded position errors in the system. From (6), it is clear that a bounded $\dot{E}_{m}$ and $E_{m}$ result in a bounded $\dot{X}_{p}$ as well. While $X_{p}$ does not explicitly enter into the error dynamics, it does affect the value of the Cartesian inertia matrices.

With $Y=\left[\begin{array}{lll}\dot{E}_{m}^{T} & E_{m}^{T} \dot{X}_{s}^{T} & E_{s}^{T}\end{array}\right]^{T}$ and $U=\left[\begin{array}{ll}F_{h}^{T} & F_{e}^{T}\end{array}\right]^{T}$, (6) can be rewritten as

$$
\left[\begin{array}{c}
\dot{Y} \\
\dot{X}_{p}
\end{array}\right]=\left[\begin{array}{cc}
A(t) & 0 \\
G(t) & 0
\end{array}\right]\left[\begin{array}{c}
Y \\
X_{p}
\end{array}\right]+\left[\begin{array}{c}
B(t) \\
0
\end{array}\right] U
$$

Then stability, as described above, can be characterized by considering the stability of

$$
\dot{Y}=A(t) Y+B(t) U
$$

We omit a stability proof here, but in [1] we developed a sufficient condition for the uniform exponential stability and BIBO stability of (8). The sufficient condition relies on $\hat{M}_{x m}\left(\Theta_{m}\right)$ in (7) being updated at a relatively slow rate - for the experimental system of Section 4, this slow update rate is $10 \mathrm{~Hz}$. In practice, we find that the matrix $\hat{M}_{x m}\left(\Theta_{m}\right)$ can be updated continuously without harming stability, but we also find that the slow update rate does not impact on performance in any perceivable way (dynamic model parameters can often be updated at slower rates than the servo rate without significant impact on performance [5]).

\subsection{System Characteristics}

In this section we analytically explore some of the distinguishing characteristics of this control system - namely, pseudo-admittance, quasi-static transparency, and steady-hand behavior. Here, pseudoadmittance explicitly refers to the mimicking of the admittance control of (1). Also recall that quasi-static transparency refers to the position and force correspondence between the master and slave at static equilibria, which is closely approximated at slow velocities, and steady-hand behavior refers to the attenuation of user hand tremor at the slave. These properties are also verified through experiment and simulation in Section 4.

We begin by considering the static equilibrium points for our system. If we assume a perfect implementation of the master's Cartesian inertia matrix $\left(\hat{M}_{x m}=M_{x m}\right)$ in the proxy dynamics, a static equilibrium is defined by $\left[\dot{Y}^{T} \dot{X}_{p}^{T}\right]^{T}=0$. Assuming $K_{a}$ is invertible, static equilibrium only occurs when $F_{h}=-\gamma_{f} F_{e}$, and the associated static equilibrium state vector is

$$
\left[\begin{array}{c}
\dot{E}_{m} \\
E_{m} \\
\dot{X}_{s} \\
E_{s} \\
X_{p}
\end{array}\right]=\left[\begin{array}{c}
0 \\
0 \\
0 \\
K_{p s}^{-1} M_{x s}^{-1}\left(\Theta_{s}\right) F_{e} \\
X_{s}-K_{p s}^{-1} M_{x s}^{-1}\left(\Theta_{s}\right) F_{e}
\end{array}\right]
$$

Thus, there is a unique static equilibrium associated with each slave position for a given $F_{e}$. At this static equilibrium, the proxy is at a position such that the user exactly feels the scaled slave/environment force $\left(F_{h}=-\gamma_{f} F_{e}\right)$ and there is perfect position correspondence between the master and the slave $\left(E_{m}=0\right)$. These two properties define the first component of quasi-static transparency. The existence of these static equilibria assumes that the applied forces are not greater than those that the robots are capable of applying. If the actuators saturate, the system loses control authority to drive $E_{m} \rightarrow 0$; in this case, the proxy position could grow unbounded as well.

To gain additional insight into the system's performance, consider the system when moving, but so slowly that $\dot{M}_{x m}\left(\Theta_{m}\right)$ and $\dot{M}_{x s}\left(\Theta_{s}\right)$ are negligible. For a given constant input vector, the equi- 
librium state of (6) is found to be

$$
\left[\begin{array}{c}
\dot{E}_{m} \\
E_{m} \\
\dot{X}_{s} \\
E_{s} \\
X_{p}
\end{array}\right]=\left[\begin{array}{c}
0 \\
-K_{p m}^{-1} M_{x m}^{-1}\left(\Theta_{m}\right)\left(F_{h}+\gamma_{f} F_{e}\right) \\
K_{a}\left(F_{h}+\gamma_{f} F_{e}\right) \\
K_{p s}^{-1}\left(M_{x s}^{-1}\left(\Theta_{s}\right) F_{e}-K_{d s} K_{a}\left(F_{h}+\gamma_{f} F_{e}\right)\right) \\
X_{s}-K_{p s}^{-1}\left(M_{x s}^{-1}\left(\Theta_{s}\right) F_{e}-K_{d s} K_{a}\left(F_{h}+\gamma_{f} F_{e}\right)\right)
\end{array}\right]
$$

For a given set of input forces, this represents the local equilibrium state associated with a given master and slave position. The values of $E_{m}$ and $E_{s}$ vary across the workspace for the same input forces, due to the effects of the underlying linearizing and decoupling controller. The master and the slave tend to move under the admittance-control paradigm of (1) as we move slowly across the workspace.

We found above that $E_{m}=0$ at static equilibrium. This is not the case when the system is moving. In fact, the position error $E_{m}$ is used to drive the movement of the system. The position error $E_{m}$ is related to the applied forces, and consequently, the velocity of the system. As we apply small input forces (that is, when the difference between $F_{h}$ and $-\gamma_{f} F_{e}$ is small), $E_{m}$ becomes small, and the system moves slowly across the workspace. This in turn leads to the steady-state velocity properties discussed above. This is the second component of quasi-static transparency: as the velocity of the system is reduced, the system approaches perfect transparency.

The above results assume that $\hat{M}_{x m}\left(\Theta_{m}\right)=M_{x m}\left(\Theta_{m}\right)$. This assumption was questioned in [1], and we found that the error in the slave velocity (i.e., the deviation from quasi-static admittance control) is proportional to the error in $\hat{M}_{x m}\left(\Theta_{m}\right)$.

It is also reasonable to wonder if a switch between controllers, due to the slow update rate of $\hat{M}_{x m}\left(\Theta_{m}\right)$ in the proxy dynamics, will create an impulse that will add noise to the system that is possibly felt by the user. An update in $\hat{M}_{x m}\left(\Theta_{m}\right)$ causes a discontinuity in the proxy velocity of (7), but it does not cause a discontinuity in the proxy position $X_{p}$. The slave controller of (2) does not rely on $\dot{X}_{p}$, so the slave's actuator does not display a discontinuity. The master servos to the slave, so it does not experience a discontinuity either. Thus, the user does not feel the switching event.

By including an $\dot{X}_{S}$ term in the master controller (3), as well as direct force feedback, we have provided a means for high-bandwidth haptic information to be relayed to the user. The slave does not servo to the proxy with PD control, but rather, with proportional control plus velocity feedback. By excluding $\dot{X}_{p}$ in (2), we create a well-damped slave without the ability to track high-frequency inputs. In addition, the integrating nature of the proxy dynamics tends to attenuate and average high-bandwidth movements of the master relative to the slave. This creates a "steady-hand" behavior in the system.

\section{Guidance Virtual Fixtures}

The Pseudo-admittance controller also enables implementation of so-called passive GVFs, which were introduced in Section 1. In this section, we extend the GVFs introduced in [2], originally designed for admittance-type human-machine cooperative systems, to Pseudo-admittance Bilateral Telemanipulation. The construction of the the Pseudo-admittance controller, specifically the use of a proxy, lends itself to this type of GVF.

Prior GVF schemes for telemanipulation have put the GVF on either the master or slave side - our method is different in that respect, in that the entire system is integrated into the implementation of the GVF. The philosophy behind previous proxy-based GVF methods has been to restrict the proxy to desired subsets of the workspace (described as "virtual fixtures," "virtual mechanisms," etc.) $[11,16]$. But why restrict the proxy to the desired path or surface, if our actual goal is to move the slave along the desired path or surface? If the slave robot experiences a disturbance load, that method could actually keep the slave off of the desired path or surface, unless the slave tracks the proxy perfectly. Our method is different from previous work in this respect; the proxy has the potential to move anywhere in the workspace, in an attempt to keep the slave moving on the desired path or surface. Allowing the proxy to move anywhere in the workspace also lends itself to GVFs that act as guidance in the truest sense of the word. Our GVF allows the user to maintain ultimate control of the system - the slave is allowed to potentially move anywhere in the workspace. Our GVF uses instantaneous preferred directions of motion to haptically assist the user in easily moving the slave along desired paths or surfaces, while imposing guarded motion when the user intentionally moves away from the desired path or surface.

In general, the desired path or surface that we would like the slave to move along may have any continuous geometry. For brevity, the remainder of this section will refer to the desired path or surface simply as the path. We assume that we can instantaneously find the point on the path that is closest to the slave - we call this point $X_{v f}$. Finding this closest point constitutes its own field of research in computational geometry, and we will not address it here. We then define the GVF error as the vector between the slave and the path:

$$
E_{v f}=X_{v f}-X_{s}
$$

The path may be instantaneously defined by a linear subspace described by a $3 \times n$ matrix $\Delta_{v f}$, where the $n$ linearly independent columns form an orthonormal basis for the path space at $X_{v f}(n=1$ for a line, $n=2$ for a plane). If the path is not continuously differentiable, additional system intelligence will be required to define $\Delta_{v f}$ at any corners.

We will consider the force that the user applies to overcome the master's PD controller, $F_{P D m}$, as the input to the GVF. Recalling (3), if the user is perfectly balancing any reflected environmental force, we have $F_{P D m}=0$, which is interpreted as no input to the GVF (i.e., no velocity command). We find the projection of the input force into the path space:

$$
F_{\Delta}=\Delta_{v f} \Delta_{v f}^{T} F_{P D m}
$$

We then construct the instantaneous unit tangent vector to the path as:

$$
\hat{T}_{v f}=\left\{\begin{array}{ccc}
\frac{F_{\Delta}}{\left\|F_{\Delta}\right\|} & : & \left\|F_{\Delta}\right\|>0 \\
0 & : & \left\|F_{\Delta}\right\|=0
\end{array}\right.
$$

We now define the preferred direction of the GVF as

$$
P_{v f}=k_{v f} E_{v f}+\hat{T}_{v f}
$$

where $k_{v f}$ is the user-defined GVF stiffness. Though this term does not have traditional stiffness units, it does determine how much the preferred direction tries to influence movement back toward the path, as a function of the GVF error $E_{v f}$. We will make use of the normalized preferred direction:

$$
\hat{P}_{v f}=\left\{\begin{array}{ccc}
\frac{P_{v f}}{\left\|P_{v f}\right\|}: & \left\|P_{v f}\right\|>0 \\
0 & : & \left\|P_{v f}\right\|=0
\end{array}\right.
$$

We next break the input force $F_{P D m}$ into components in the preferred direction

$$
F_{P}=\left\{\begin{array}{cll}
\left(\hat{P}_{v f}^{T} F_{P D m}\right) \hat{P}_{v f} & : & \hat{P}_{v f}^{T} F_{P D m}>0 \\
0 & : & \hat{P}_{v f}^{T} F_{P D m} \leq 0
\end{array}\right.
$$

and in the nonpreferred directions

$$
F_{\bar{P}}=F_{P D m}-F_{P}
$$




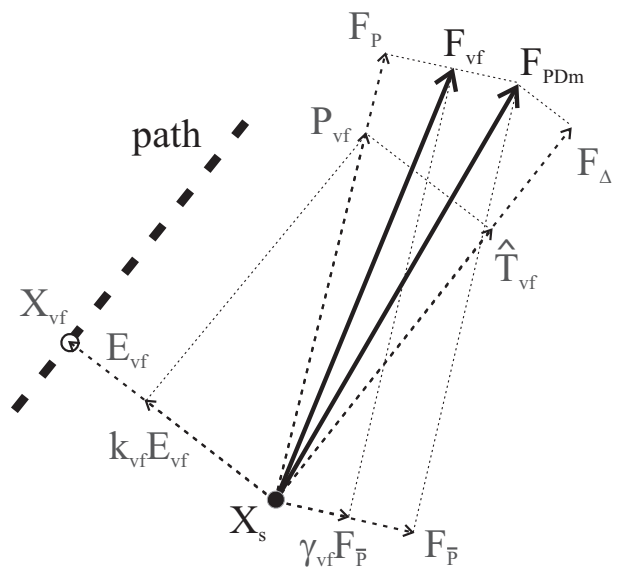

(a)

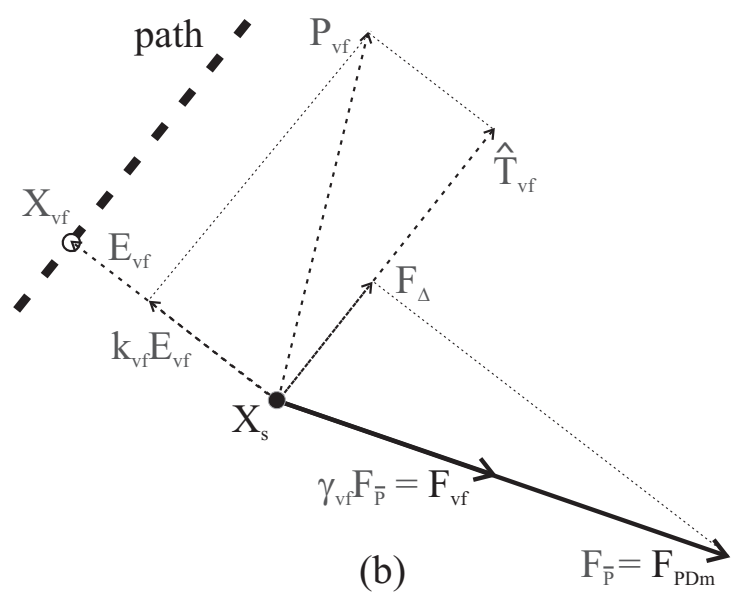

Figure 2: A geometrical view of guidance virtual fixtures when there (a) is and (b) is not a component of the applied force in the preferred direction. The virtual fixture maps the input force $F_{P D m}$ into the virtual fixture force $F_{v f}$, which is then used as a command to the proxy.

We then construct the GVF force by combining the force in the preferred direction with an attenuation of the force in the nonpreferred directions:

$$
F_{v f}=F_{P}+\gamma_{v f} F_{\bar{P}}
$$

where $\gamma_{v f} \in[0,1]$ is the user-defined GVF attenuation gain. To implement the GVF, we modify the proxy dynamics of (4) to

$$
\dot{X}_{p}=k_{a} F_{v f}
$$

where $k_{a}$ is a scalar admittance gain.

Figure 2 provides a geometric depiction of the GVF. If there is a component of the applied force $F_{P D m}$ in the preferred direction, the algorithm maps the applied force into the GVF force $F_{v f}$, which points more in the direction of the path than did the original. This moves the proxy in a direction that will tend to move the slave (which is servoing to the proxy) towards the path. The applied force is slightly attenuated in magnitude in creating $F_{v f}$. If there is no component of the applied force $F_{P D m}$ in the preferred direction, the GVF does not change the direction of the applied force, but the
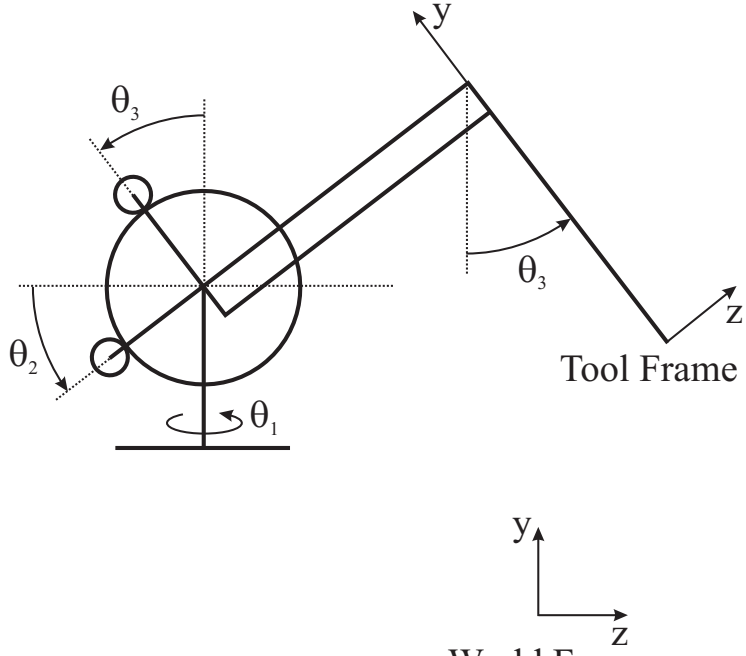

World Frame

Figure 3: PHANToM frame description, assuming right-handed coordinate systems. The tool frame coincides with the world frame at the zero position of the PHANToM.

magnitude is attenuated by $\gamma_{v f}$. Thus, if the user commands a force that is intentionally moving away from the path, the GVF does not guide the user towards the path, but rather, it forces the user to move in a guarded fashion.

\section{EXPERIMENTAL VERIFICATION}

In this section we verify the properties of Pseudo-admittance control, with and without GVFs, through both simulations and experiments. Our experimental system is constructed of two PHANToM robots from SensAble Technologies [15], run on a single computer at a sampling rate of $500 \mathrm{~Hz}$. In addition to the experimental system, we have constructed a MATLAB simulation of our system. This allows us to explore certain aspects of system performance in a more controlled setting. By synthesizing two prior works $[4,6]$, we implemented an adaptive algorithm, run off-line, to obtain the necessary PHANToM parameters for the control system and the simulation; details can be found in [1]. To obtain the measurement of the environmental force $F_{e}$ in our experimental setup, we use the force generated at the tool tip by the slave's actuators as an approximation of the true force. Figure 3 shows the PHANToM coordinate system frames used throughout this section.

The human user is an integral component in this human-machine collaborative system, but human motions and physical parameters are also highly variable. We have created a simulation for our PHANToM telemanipulation system that allows us to obtain less anecdotal results than we would obtain from experiments alone. We make use of our simulation now to consider, in a controlled fashion, the behaviors of the Pseudo-admittance Bilateral Telemanipulation system when interacting with various types of environments.

The top of Fig. 4 shows how our system interacts with purely viscous environments. We include damping $b_{e}=0 \mathrm{Ns} / \mathrm{m}$, which is the slave moving freely in space. For each simulation, the PHANToMs begins in the zero position, embedded in the simulated environment. The user applies a constant force $F_{h}$ in the $x$ direction of the world frame for the first three seconds, and then releases the device $\left(F_{h}=0\right)$. The plot shows the position in the $x$ direction of the world frame. From these plots, the nature of the Pseudo-admittance controller becomes clear. The slave moves with a velocity that is almost perfectly linearly proportional to the applied force. The position er- 

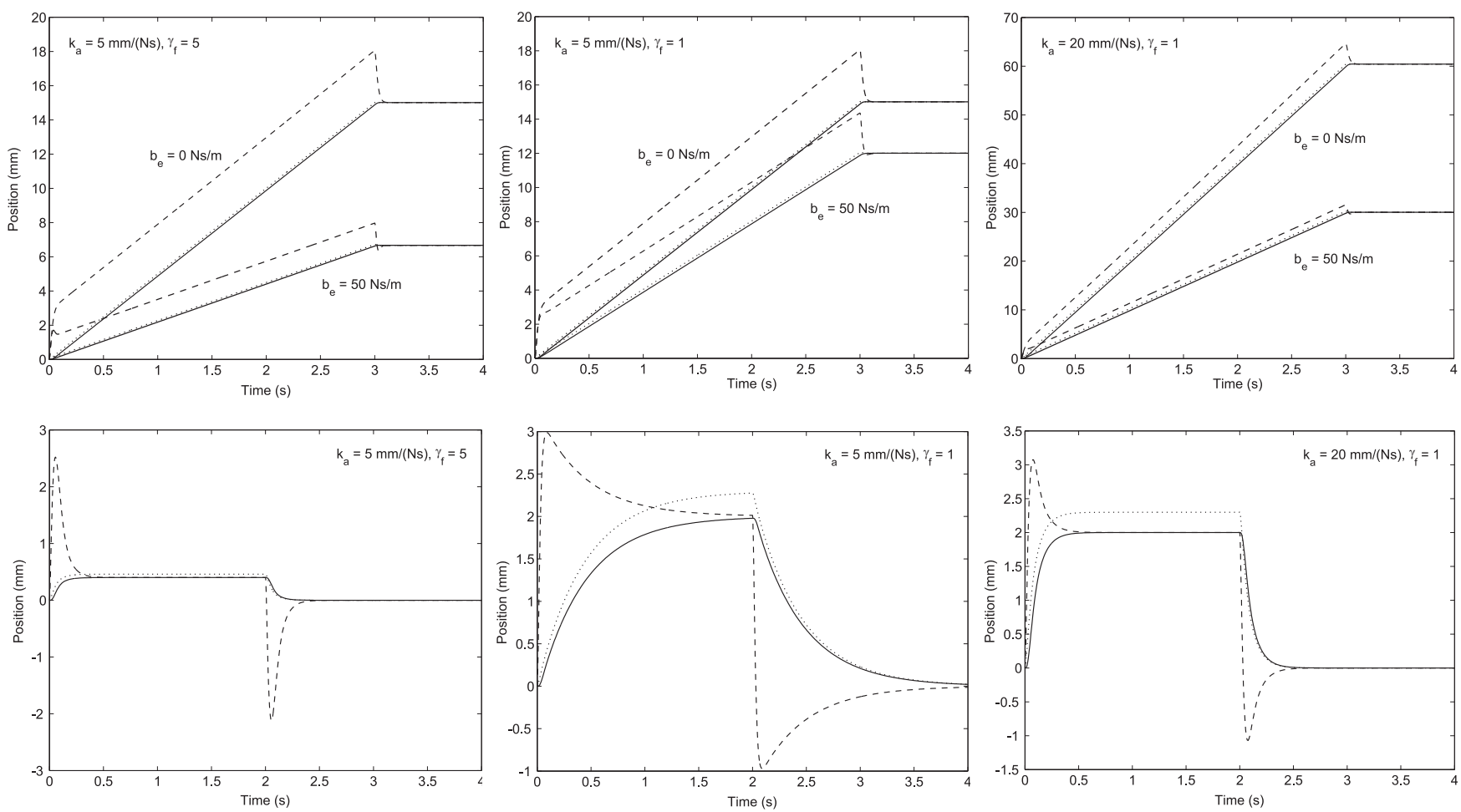

Figure 4: Simulated interactions with (top) viscous environments with damping $b_{e}$ and (bottom) elastic environment with spring constant $k_{e}=500 \mathrm{~N} / \mathrm{m}$. Master (- ), slave (-), and proxy $(\cdots)$ trajectories are shown. The user applies a constant force $F_{h}=1 \mathrm{~N}$ for the first three seconds, and then releases the master.

ror between the master and slave is used to drive that movement. When the master is released, the position error disappears, and the system stays where it was released by the user.

The bottom of Fig. 4 shows how our system interacts with purely elastic environments. We simulate a unilateral spring surface that pushes in the $-x$ direction of the world frame when the slave enters the $+x$ half-space. For each simulation, the PHANToM begins in the zero position, at the surface of the simulated environment. The user applies a constant force $F_{h}$ in the $+x$ direction of the world frame for the first two seconds, and then releases the device $\left(F_{h}=0\right)$. The plot shows the position in the $x$ direction of the world frame. It is evident that a constant input force $F_{h}$ does result in a static equilibrium with an elastic environment. In addition, the position error between the master and the slave vanishes. The proxy reaches into the environment, pulling the slave behind it, until the force generated is large enough to drive the master back into static equilibrium. When the master device is released with potential energy stored in the environment, the environment pushes the slave out to the surface. The master is temporarily pushed well outside the boundaries of the environment by the force-feedforward term, but the position error between the master and the slave eventually vanishes, with the system coming to rest just outside the elastic environment. In practice, a dead-man switch should be used to only allow proxy movement when the user is holding the master.

We begin our experiments by demonstrating the steady-hand properties of Pseudo-admittance control. Figure 5 shows the master and proxy positions along two coordinate axes of the world frame as the user moves the telemanipulator across the workspace. The slave is moving in free space, and it servos to the proxy. The user displays hand tremor that is greatly attenuated in the proxy. Also, at approximately the 5-second mark, the user releases the master, and the system stays in place, confirming the stability of the system.
Next we consider the implementation of a basic GVF, a vertical plane, on our experimental system. The plane is defined by $x=0$ in the world frame. Figure 6 shows GVFs implemented with two different $k_{v f}$ values. In each plot, we start at rest (at four different initial conditions) near the bottom of the plot. Then the user simply applies a gentle force in approximately the positive $y$ direction of the world frame. The plots show the resulting movement of the master device, as well as the proxy (to which the slave servos). It is evident from the figure how increasing $k_{v f}$ points the preferred direction more towards the desired plane than parallel to it. Reducing $\gamma_{v f}$ has a similar but smaller effect by attenuating the component of the input force, and consequently the commanded velocity, in the the non-preferred directions. The benefits of these GVFs are evident; the device closely approaches the desired surface with essentially no cognitive effort on the part of the user. Recall that the user has complete control to move the device away from the plane at all times; these are simply the paths that the robots favor.

\section{CONCLUSIONS AND Future WORK}

In this paper, we presented a novel bilateral telemanipulation control system called Pseudo-admittance. This system is designed to mimic admittance control on systems where the master is an impedance-type robot. It has many desirable properties, such as steady-hand tremor attenuation, quasi-static transparency, and the ability to include guidance virtual fixtures. A novel guidance virtual fixture method was presented that builds upon a method previously developed for human-machine cooperative systems. The properties of Pseudo-admittance Bilateral Telemanipulation, with and without guidance virtual fixtures, were verified through simulations and experiments on a system where both the master and 

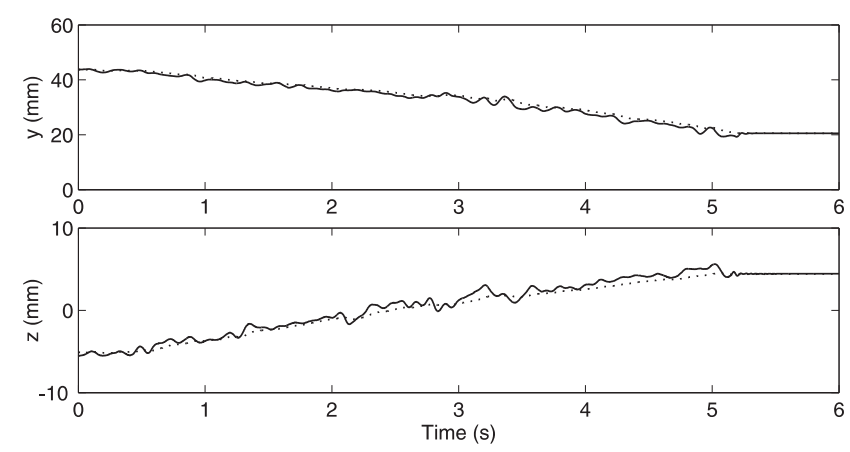

Figure 5: An experimental demonstration of the steady-hand behavior seen in Pseudo-admittance control $\left(k_{a}=30 \mathrm{~mm} /(\mathrm{Ns})\right)$. Master $(-)$ and proxy $(\cdots)$ trajectories are shown.

slave are of the impedance-type. Pseudo-admittance has potential benefits for systems that are designed for stability and transparency, but that require better-than-human levels of precision during the execution of certain tasks. Pseudo-admittance could also be applied to systems with large motion scaling or velocity limits at the slave - systems which are typically run under rate control. Guidance virtual fixtures could be used as task macros - potentially increasing both speed and precision on structured tasks that still require direct human control.

Qualitatively, this system feels very stable - it feels almost like a pure, high viscosity. The system interacts well with every environment (including rigid steel) for a large range of admittance gain values. While the control system does not exhibit transparency in the traditional sense, it does provide the user with a rich set of haptic information. The system is specifically designed to assist the user with precise, slow-moving tasks, and consequently the system provides the best sense of telepresence for the user when commanded to move at slow velocities.

A remaining question is how best to control orientation of the end effector under Pseudo-admittance control. Because of the noncommuting nature of rotations, it is not clear if Pseudo-admittance translates to orientation. However, position does not scale with orientation like it does with rectilinear motion; a full turn of the end effector is always $2 \pi$ radians. For systems with large motion scaling, it may be desirable to implement Pseudo-admittance control on the positioning stage of the robot, but another method for orientation. This topic is left as future work.

Finally, in this paper we were able to analytically show BIBO stability of our system. However, this is not sufficient to prove stability of the system when coupled to arbitrary users and environments. Additional stability techniques, such as passivity, could be considered in future work.

\section{REFERENCES}

[1] J. J. Abbott. Virtual Fixtures for Bilateral Telemanipulation. PhD thesis, Department of Mechanical Engineering, The Johns Hopkins University, 2005.

[2] A. Bettini, P. Marayong, S. Lang, A. M. Okamura, and G. D. Hager. Vision-assisted control for manipulation using virtual fixtures. IEEE Trans. Robotics, 20(6):953-966, 2004.

[3] A. Casals, L. Munoz, and J. Amat. Workspace deformation based teleoperation for the increase of movement precision. In Proc. IEEE Int'l. Conf. on Robotics and Automation, pages 2824-2829, 2003.

[4] M. C. Çavuşoğlu, D. Feygin, and F. Tendick. A critical study of the mechanical and electrical properties of the PHANToM haptic interface and improvements for high-performance conrol. Presence, 11(6):555$568,2002$.
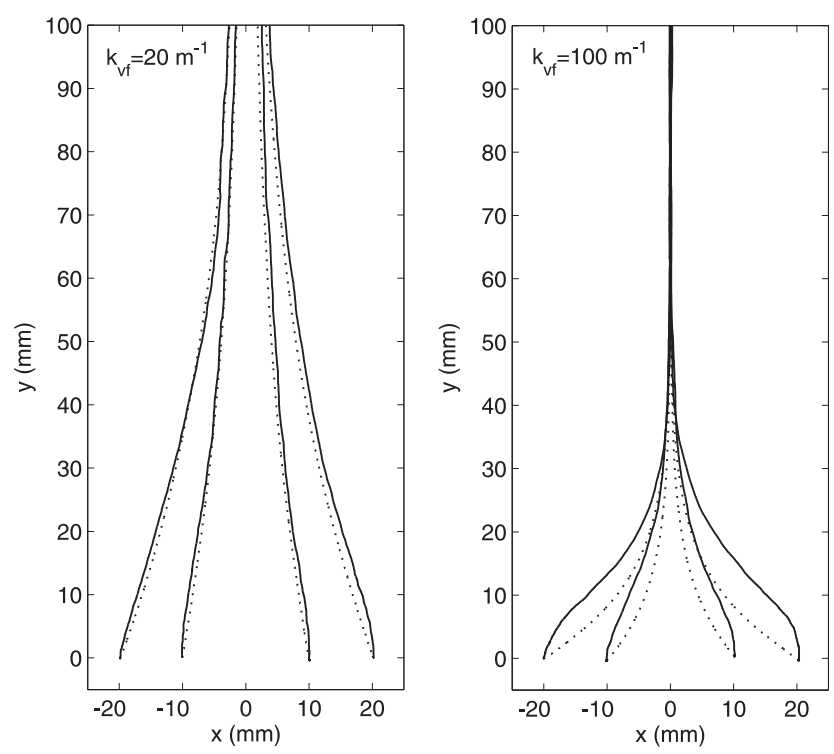

Figure 6: Experimental data showing guidance virtual fixtures for the vertical plane defined by $x=0$. Master (-) and proxy $(\cdots)$ trajectories are shown. Data is shown for $\gamma_{v f}=0.05$ and $k_{a}=40$ $\mathrm{mm} /(\mathrm{Ns})$.

[5] J. J. Craig. Introduction to Robotics: Mechanics and Control. Addison Wesley, Reading, Massachusetts, second edition, 1989.

[6] J. J. Craig, P. Hsu, and S. S. Sastry. Adaptive control of mechanical manipulators. Int'l. J. Robotics Research, 6(2):16-28, 1987.

[7] Intuitive Surgical. http://www.intuitivesurgical.com.

[8] W. S. Kim, F. Tendick, S. R. Ellis, and L. W. Stark. A comparison of position and rate control for telemanipulators with consideration of manipulator system dynamics. IEEE J. Robotics and Automation, RA-3(5):426-436, 1987.

[9] M. E. Kontz and W. J. Book. Position/rate haptic control of a hydraulic forklift. In Proc. ASME Int'l. Mechanical Engineering Congress and Exposition, pages 801-808, 2003.

[10] P. D. Lawrence, S. E. Salcudean, N. Sepehri, D. Chan, S. Bachmann, N. Parker, M. Zhu, and R. Frenette. Coordinated and force-feedback control of hydraulic excavators. In Proc. 4th Int'l. Symposium on Experimental Robotics, pages 181-194, 1995.

[11] A. Micaelli, C. Bidard, and C. Andriot. Decoupling control based on virtual mechanisms for telemanipulation. In Proc. IEEE Int'l. Conf. on Robotics and Automation, pages 1924-1931, 1998.

[12] F. Mobasser and K. Hashtrudi-Zaad. Implementation of a rate mode impedance reflecting teleoperation controller on a haptic simulation system. In Proc. IEEE Int'l. Conf. on Robotics and Automation, pages 1974-1979, 2004.

[13] C. A. Moore, M. A. Peshkin, and J. E. Colgate. Cobot implementation of virtual paths and 3-D virtual surfaces. IEEE Trans. Robotics and Automation, 19(2):347-351, 2003.

[14] J. Roy and L. L. Whitcomb. Adaptive force control of position/velocity controlled robots: Theory and experiment. IEEE Trans. Robotics and Automation, 18(2):121-137, 2002.

[15] SensAble Technologies. http://www.sensable.com.

[16] N. Turro, O. Khatib, and E. Coste-Maniere. Haptically augmented teleoperation. In Proc. IEEE Int'l. Conf. on Robotics and Automation, pages 386-392, 2001.

[17] R. L. Williams II, J. M. Henry, M. A. Murphy, and D. W. Repperger. Naturally-transitioning rate-to-force control in free and constrained motion. ASME J. Dynamic Systems, Measurement, and Control, 121(3):425-432, 1999.

[18] C. B. Zilles and J. K. Salisbury. A constraint-based god-object method for haptic display. In Proc. IEEE/RSJ Int'l. Conf. on Intelligent Robots and Systems, pages 146-151, 1995. 